\title{
Clinical, Epidemiological and Socio-Personal Characteristics of Patients with Sexually Transmitted Infections in the Republic of Sakha (Yakutia)
}

\author{
Lubov I. Petrova, $\mathrm{PhD}^{1}$; Snezhana S. Sleptsova, $\mathrm{PhD}, \mathrm{ScD}^{1^{*}}$; Spiridon S. Sleptsov, $\mathrm{PhD}^{2}$ \\ ${ }^{1}$ M. K. Ammosov North-Eastern Federal University \\ ${ }^{2}$ Yakut Science Center of Complex Medical Problems \\ Yakutsk, the Republic of Sakha (Yakutia), Russia
}

\begin{abstract}
Background: The spread of sexually transmitted infections (STIs) among the population of the Russian Federation, as well as throughout the world, today remains an urgent problem for health care in general and for doctors of various specialties. The importance of the problem of STIs is caused not only by their distribution, but also by the adverse consequences in the form of severe complications, such as infertility, pathology of pregnancy and childbirth. The purpose of this study was to justify and develop medical and social health measures aimed at improving the health indicators of young STI patients based on a comprehensive socio-hygienic study of their health status and lifestyle, according to information from the Yakut Republican Dermato-Venerologic Dispensary.

Materials and Methods: In order to obtain representative results in the study of health status, socio-hygienic characteristics of lifestyle, and organization of medical care under supervision, 485 patients with STIs, aged between 18 and 29 years, were included in the main group. To determine the factors contributing to the occurrence of STIs among the population, the control group $(\mathrm{n}=415)$ of healthy people was formed.

Results: The results obtained lead to the conclusion that the main age group at risk for STIs is the 25-29 group. Patients with chronic forms of STIs are more likely to have complications in the form of various inflammatory diseases of the urogenital sphere. Marital status is characterized by the absence of strong marital relations the majority of STI patients had multiple and casual sex. Among this cohort of patients, alcohol consumption was more often recorded. The incidence of young STI patients was influenced by unfavorable socio-hygienic lifestyle factors, among which the most significant were low medical behavior (irregular visits to doctors and implementation of their recommendations), poor family relationships (conflicts with relatives), bad habits (alcohol abuse, drug addiction), and low awareness of STI prevention.

Conclusion: Successful recovery of health is largely determined by the implementation of rehabilitation measures. Medical and social rehabilitation of STI patients should be based on medical, social and psychological measures. (International Journal of Biomedicine. 2019;9(3):266-268.)
\end{abstract}

Key Words: sexually transmitted infections $\bullet$ youth $\bullet$ sexual behavior $\bullet$ awareness $\bullet$ morbidity $\bullet$ prevention

\section{Introduction}

The spread of sexually transmitted infections (STIs) among the population of the Russian Federation, as well as throughout the world, today remains an urgent problem for health care in general and for doctors of various specialties. The importance of the problem of STIs is caused not only by their distribution, but also by the adverse consequences in the form of severe complications, such as infertility, pathology

*Corresponding author: Prof. Snezhana S. Sleptsova, MD PhD, ScD. M. K. Ammosov North-Eastern Federal University. Yakutsk, the Sakha Republic, Russia. E-mail: sssleptsova@yandex.ru of pregnancy and childbirth. Violation of the reproductive function of citizens is one of the reasons for the decline in fertility, which in general can affect population growth, leading to a worsening of the demographic situation in the country as a whole. ${ }^{(1,2)}$ Given the current situation, it is necessary to search for new approaches to organizing and improving medical and social care for young people with STIs, which requires a detailed study of their health characteristics and the identification of factors that currently affect the occurrence and adverse course of STIs. ${ }^{(3,4)}$

The purpose of this study was to justify and develop medical and social health measures aimed at improving the health indicators of young STI patients based on a 
comprehensive socio-hygienic study of their health status and lifestyle, according to information from the Yakut Republican Dermato-Venerologic Dispensary.

\section{Materials and Methods}

In order to obtain representative results in the study of health status, socio-hygienic characteristics of lifestyle, and organization of medical care under supervision, 485 patients with STIs, aged between 18 and 29 years, were included in the main group (MG). To determine the factors contributing to the occurrence of STIs among the population, the control group (CG) $(n=415)$ of healthy people was formed. Statistical analysis was performed using SPSS (version 19.0). Baseline characteristics were summarized as frequencies and percentages for categorical variables. Group comparisons with respect to categorical variables were performed using the Chi square test. A probability value of $P<0.05$ was considered statistically significant.

\section{Results and Discussion}

The results of a study of the incidence of sexually transmitted diseases among young patients with STIs showed that trichomoniasis accounts for the bulk of the structure of STIs (40.1\%). Next was gonorrhea (up to $24.1 \%$ ), followed by syphilis (18.5\%), chlamydia (10.9\%), anogenital warts (4.1\%), and urogenital herpes $(2.3 \%)$. This structure was significantly different among men and women. Thus, among men, the leading diseases were gonorrhea $(69.3 \%$,), anogenital warts $(65 \%)$, and chlamydial infection $(60.3 \%)$. Among women, the majority of cases of STIs belonged to trichomoniasis (84.1\%).

The structure of morbidity differed among persons of different ages. In the age group of 18-20 years, the proportion of gonorrhea and anogenital warts was highest. The proportion of syphilis and urogenital herpes was maximal at the age of 2124, trichomoniasis and chlamydial infection - at 25-29 years.

The highest rate of syphilis among men occurred in the age groups 18-20 years and 21-24 years, of gonorrhea - among men in all age groups, chlamydial infection in the 18-20 group $(P<0.05)$, urogenital herpes in the $25-29$ group $(P<0.05)$, and anogenital warts in the 18-20 group $(P<0.05)$. Among women of all age groups, the rate of patients with trichomoniasis was 5.3 times higher $(P<0.05)$ than among men $(84.1 \%$ vs. $15.9 \%)$.

The main age group at risk is the age of 25-29 years, which accounts for the majority of STI patients (42.5\%). More than half of the patients in this age group had trichomoniasis and chlamydial infection. In the second place in the structure of the diseased were persons aged between 21 and 24 years $(36.3 \%)$, where syphilis and genital herpes predominate. The third place belongs to the age group of $18-20$ years $(21.2 \%)$; a significant percentage of patients in this age group had gonorrhea and anogenital warts.

The study revealed the presence of complications, depending on the course of STIs. In patients with chronic forms of STIs with a longer course of the disease, compared with patients with acute forms of STIs, there were 8.1 times $(P<0.05)$ more frequent complications in the form of various inflammatory diseases of the urogenital sphere $(39.6 \%$ vs. $4.9 \%$ ) and 13 times more frequent extragenital lesions (joint damage in the form of gonorrheal and chlamydial arthritis) $(15.6 \%$ vs. $1.2 \%, P<0.05)$.

The study of the socio-hygienic characteristics of STI patients of young working age revealed that persons aged 2529 years $(42.5 \%)$ make up a large part; the second and third ranking places $(36.3 \%$ and $21.2 \%)$ belong to the age groups 21-24 and 18-20 years, respectively.

Among patients of $\mathrm{MG}$, compared to $\mathrm{CG}$, there was a prevalence of secondary special education: $18.3 \%$ had a secondary education, and $9.4 \%$ had higher education; students of secondary special and higher educational institutions made up $17.6 \%$. In CG, 34.5\% had higher education, $37.9 \%$ had secondary special and higher education, and $2.9 \%$ had secondary education.

The marital status in MG was characterized by the absence of strong and stable marital relations. Only $12.8 \%$ of patients were married, which was 3.2 times lower than in CG (41.4\%), $14.7 \%$ indicated that they were in a civil marriage versus $2.1 \%$ in CG, and $11.4 \%$ were divorced. Single, never married, in both groups were distributed almost equally: $61.1 \%$ and $56.5 \%$, respectively.

In $\mathrm{MG}$, drug use was more commonly reported. Thus, in $\mathrm{MG}$, compared to $\mathrm{CG}$, alcohol abusers were $23.6 \%$ versus $1.3 \%(P<0.05)$. The largest percentage of the first use of alcoholic beverages in MG falls in the age period of 9-11 years (17-20 years in CG). About $23.6 \%$ of persons drank alcohol almost every day, $58.8 \%$ once a week, and $17.6 \%$ on holidays. In CG: every day $-1.3 \%$, once a week $-33.5 \%$, on holidays $39.2 \%$, 2-3 times a year - 9\%, do not use at all - 17\%. Alcohol consumption was most often observed at the age of 24-29 years $(57.9 \%$ in $\mathrm{MG}$ and $56.6 \%$ in $\mathrm{CG})$. In $\mathrm{MG}, 4.2 \%$ of patients were registered in the narcological dispensary; there were none in CG.

According to the results of a questionnaire survey, in MG narcotic substances were regularly consumed 10.5 times more often $(P<0.05)$ than in CG $(16.8 \% v s .1 .6 \%)$. Of these, smoking with a content of narcotic substances took the first place $(51.6 \%)$, then came the use of tableted drugs (39.4\%) and injected drugs (9\%). The first use of the drug in $81.4 \%$ patients was noted at the age of 17-18 years (the main motive was curiosity in $73.1 \%$ cases).

Analysis of sexual behavior and individual attitudes among STI patients showed that $68.3 \%$ had the first sexual contact at age of $15-17$ years versus $18.3 \%$ in CG. During the last six months, only $39.6 \%$ patients in MG had one sexual partner vs. $74.8 \%$ persons in CG. The majority $(60.4 \%)$ of STI patients had multiple ( 3 and more) and casual sexual contacts.

The study of value judgments regarding possible sexual practices showed that in the main group, $42.4 \%$ would have allowed themselves random relationships, in the control group $38.8 \%$; premarital relations in $\mathrm{MG}-57.6 \%$ and in $\mathrm{CG}-62 \%$; extramarital relations in $\mathrm{MG}-78 \%$, in $\mathrm{CG}-47.6 \%$.

The study of awareness of STI prevention showed that the main source of information in both groups was conversations with peers $(50.4 \%$ in $\mathrm{MG}$ and $44.4 \%$ in $\mathrm{CG})$, followed by popular literature $(20.4 \%$ and $32.4 \%$, respectively), and 
only $18.4 \%$ and $15.6 \%$ received information from doctors. Considering the sources of the information received, which were the peers and the "yellow press," suggests that the information obtained was superficial and not specific.

\section{Conclusion}

The results obtained lead to the conclusion that the main age group at risk for STIs is the 25-29 group. Patients with chronic forms of STIs are more likely to have complications in the form of various inflammatory diseases of the urogenital sphere. Marital status is characterized by the absence of strong marital relations the majority of STI patients had multiple and casual sex. Among this cohort of patients, alcohol consumption was more often recorded.

The incidence of young STI patients was influenced by unfavorable socio-hygienic lifestyle factors, among which the most significant were low medical behavior (irregular visits to doctors and implementation of their recommendations), poor family relationships (conflicts with relatives), bad habits (alcohol abuse, drug addiction), and low awareness of STI prevention.

Successful recovery of health is largely determined by the implementation of rehabilitation measures. Medical and social rehabilitation of STI patients should be based on medical, social and psychological measures. The basis of social rehabilitation is the introduction of elements of a healthy lifestyle, which is currently the most relevant and effective of the preventive measures that should be actively used by STI patients to correct, strengthen and restore health. The implementation of this direction should be based on increasing the level of medical literacy and giving up bad habits. The crucial point in implementing a healthy lifestyle is that patients have a responsible attitude towards their own health and the health of those around them.

\section{Competing Interests} interests.

The authors declare that they have no competing

\section{References}

1. Kisin VI, Zabirova KI, Guschina AE. Management of Patients patients with sexually transmitted infections. M.: GEOTAR-Media; 2017. [In Russian].

2. Akovbyan VA, Prohorenkov VI, Sokolovsky EB. Sexually transmitted infections. M.: Media Sfera; 2007. [In Russian].

3. Vasil'ev MM, Toskin IA. [The effectiveness of information and medical services in assisting vulnerable groups of the population in relation to sexually transmitted infections]. Vestnik Dermatologii i Venerologii. 2006;(5):44-50. [Article in Russian].

4. Kuznecova YuN, Kungurov NV. [Awareness of young people and adolescents on sexual life and sexually transmitted infections]. Russian Journal of Skin and Venereal Diseases. 2007;(6):41-45. [Article in Russian]. 